\title{
Effects of home visits on quality of life among older adults: a systematic review protocol
}

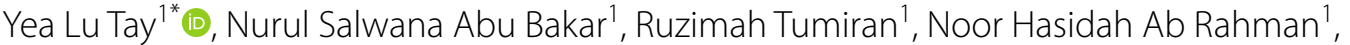 \\ Noor Areefa Ameera Mohd Ma'amor ${ }^{2}$, Weng Keong Yau ${ }^{3}$ and Zalilah Abdullah'
}

\begin{abstract}
Background: Home visiting services for older adults have been offered for decades to maintain and promote health and independent functioning, thus enhancing quality of life. Previous systematic reviews have provided a mixed picture of the benefits of home visiting programmes in older adults, primarily because of heterogeneity in study designs, targeted populations, and intervention strategies. These reviews may also become out of date; thus, an updated synthesis of relevant studies is warranted. Our objective is to perform a systematic review of recently published primary studies on the effectiveness of multi-professional home visits on quality of life among older adults.
\end{abstract}

Methods: We will perform a comprehensive search for studies investigating the effect of a multi-professional home visit approach on quality of life among older adults. We will conduct the literature search in selected electronic databases and relevant research websites from January 2010 onwards. We will include randomised controlled trials (RCTs), cluster randomised controlled trials (cluster RCTs), and observational studies that enrolled older adults without dementia over 60 years old, along with studies involving multi-professional preventive-promotive home visit approaches not related to recent hospital discharge. We will report our planned review following the Preferred Reporting Items for Systematic Reviews and Meta-Analyses (PRISMA) guidelines. We will retrieve and record relevant data in a standardised data extraction form and evaluate the quality of the included articles using the Cochrane risk of bias tool and the quality assessment tool for studies with diverse designs (QATSDD). Where appropriate, outcomes will be pooled for meta-analysis using a random-effects model. The main outcomes include quality of life, incidence of falls, depression, dementia, and emergency department admissions.

Discussion: This review may provide evidence for the effectiveness of home visits in improving older adults' quality of life. It will potentially benefit health care professionals, policymakers, and researchers by facilitating the design and delivery of interventions related to older generations and improve service delivery in future.

Systematic review registration: PROSPERO CRD42021234531.

Keywords: Systematic review, Meta-analysis, House calls, Home visits, Aged

*Correspondence: tay.yl@moh.gov.my

${ }^{1}$ Institute for Health Systems Research, National Institutes of Health, Ministry of Health Malaysia, 40170 Shah Alam, Selangor, Malaysia Full list of author information is available at the end of the article

\section{Background}

Population ageing is a global phenomenon. Most individuals expect to live into their sixties and beyond. The world population of adults aged 60 years and over is expected to nearly double from 12 to $22 \%$ between 2015 and 2050 [1]. In Malaysia, 20\% of the total population will original author(s) and the source, provide a link to the Creative Commons licence, and indicate if changes were made. The images or other third party material in this article are included in the article's Creative Commons licence, unless indicated otherwise in a credit line to the material. If material is not included in the article's Creative Commons licence and your intended use is not permitted by statutory regulation or exceeds the permitted use, you will need to obtain permission directly from the copyright holder. To view a copy of this licence, visit http://creativecommons.org/licenses/by/4.0/. The Creative Commons Public Domain Dedication waiver (http://creativeco mmons.org/publicdomain/zero/1.0/) applies to the data made available in this article, unless otherwise stated in a credit line to the data. 
be over 65 years old by 2030 [2]. A recent study indicated that the life expectancy of Malaysian older adults aged 65 years was 79.8 and 82.1 years for males and females, respectively [3]. Despite the national census statistics defining older adults as those over the age of 65, Malaysia adopted the United Nations' definition, classifying older adults as those aged 60 years and above for policy development regarding the older adult population [4]. The older adult population has a multitude of health needs and challenges, along with a deteriorating quality of life (QoL) [5].

According to the World Health Organization (WHO), QoL refers to "an individual's perception of life in the context of the culture and value system in which he or she lives and in relation to his or her goals, expectations, standards, and concerns" [6]. QoL linked to health concepts is defined as the value assigned to the duration of life, modulated by limitations, functional status, perceptions, and social opportunities, which are influenced by diseases, injuries, treatments, and health policies [7]. QoL is increasingly recognised as a focus for healthcare service delivery in the older adult population. It allows the healthcare providers and policymakers to measure the efficacy of health interventions and evaluate multisectoral public policies, which include health, social, community, and policy actions [8].

Numerous healthcare interventions have been designed and implemented with the goal of maintaining or improving QoL among older adults, and most studies indicate the importance of active ageing. These studies have demonstrated that QoL among older adults can be enhanced through low-cost interventions, such as physical exercise [9-11]. Besides, older adults utilising the home visiting services were shown to have a better QoL outcome [12, 13].

Home visits are defined as visits to an individual's home by professionals, which may include nurses, social workers, physicians, physiotherapists, occupational therapists, pharmacists and other specialists [14]. There are five types of home visiting services: palliative, rehabilitative, long-term maintenance, therapeutic, and preventivepromotive home visits [15]. Preventive-promotive home visiting services have been offered for decades with the goal of maintaining and promoting the health and independent functioning of older adults. In addition, these services aim to reduce admission to hospitals or nursing homes and the associated economic burden [16, 17].

Home visits allow health professionals to evaluate possible problems in the living environment of homebound older adults, assess their physical and mental health status, provide older adults with professional support, and refer them to specialist care if needed [17]. By reducing the risk of functional deterioration, these strategies are primarily structured to enhance the health-related QoL (HRQoL) of older adults, increase the possibility of continued independent living, and delay mortality [18].

Home visits have been shown to positively affect patient care and provider attitudes as well as increased satisfaction among homebound older adults and providers [19]. A previous study demonstrated that preventive home visits may have positive effects on QoL of older adults [20]. However, the variability in the study designs, participants, and outcome measures has made comparisons difficult. Liimata et al. (2019) conducted a randomised controlled trial (RCT) measuring the effects of preventative multidisciplinary home visits on HRQoL of older adults living independently. The team, which consisted of a nurse, a physiotherapist, and a social worker, observed a significantly slower decline of HRQoL in the intervention group, but this effect diminished after the visits ended [20]. In a separate publication from the same study, preventive home visits resulted in an improved HRQoL without incurring additional healthcare costs [21]. An effective prevention method aids in supporting quality of life among older adults. In a review on preventive home visits for older adults, Mayo-Wilson et al. (2014) analysed 64 RCTs involving older adults without dementia from database inception until December 2012. The study yielded high-quality evidence for decreasing falls but low-quality evidence for quality of life [22]. Thus, although an RCT demonstrated promising results on home visits, a review of multiple RCTs failed to observe significant results. In addition, although multi-professional preventive home visit approaches with thorough evaluation and collaboration among healthcare professionals may be more beneficial than home visits by a single professional, few studies have focused on this multi-professional preventive home visit approach [20, 23, 24].

Multi-professional preventive home visit interventions involve coordination between several health care professionals towards shared goals. Effective communication among the team members is crucial when the members work within the boundaries of their expertise and subsequently discuss progress in group sessions [25]. Previous systematic reviews have provided a mixed picture of the benefits of multi-professional home visiting services for older adults. Stuck et al. [26] and Touringy et al. [14] suggested that the multi-professional approach with follow-up visits was effective in identifying the needs of the older adult population. However, Mayo-Wilson et al. [22] demonstrated the challenges of concluding that preventive home visits result in reliable benefits, primarily due to variability in the study designs, participants, and intervention strategies of the preventive home visits approach. 


\section{Rationale}

In Malaysia, home visiting services for the older adult population are delivered by a multidisciplinary team and are primarily provided by the Ministry of Health [27]. The home visiting services offered in Malaysia include home-based treatment, pharmacy counselling, rehabilitation, and palliative services, which aim to ensure continuity of care at home, reduce hospital readmission, and improve QoL $[28,29]$. According to the National Health and Morbidity Survey (NHMS) 2018, a national community survey for elderly health in Malaysia, $28.6 \%$ of older adults perceived themselves as having poor QoL, $14.1 \%$ reported having at least one fall in the 12 months prior to the survey, $8.5 \%$ were diagnosed with dementia, and $11.2 \%$ were at risk of experiencing depressive symptoms [30]. Poor QoL in Malaysian older adults was found to be associated with lower education, depression, food insecurity, reduced functional status, and a lack of social support [31]. Hence, we seek to examine preventive-promotive strategies that specifically prevent or reduce the risk of developing dementia, depression, and falls, with the ultimate aim of improving QoL among the older adult population.

To our knowledge, the most recent systematic review of primary studies examining the multi-professional preventive home visit approach for older adults included studies conducted up to December 2012 [22]. Because the older adult population is rapidly growing, the number of studies describing the home visit intervention is increasing, and the methodological and reporting quality of these studies is improving. Hence, a comprehensive systematic review which includes recent studies is needed to provide new evidence on the effectiveness of multi-professional preventive-promotive home visits in improving QoL among older adults. This review may serve as a guideline for the healthcare professionals, policymakers, researchers, and institutions in designing and delivering interventions for older adults in future. Aligning health systems with the needs of the older adult population may help to promote healthy ageing in Malaysia in the long term.

\section{Objective}

This study aims to systematically assess the effect of a multi-professional home visit approach on QoL among older adults.

\section{Methods}

The present protocol has been registered within the PROSPERO database (registration number CRD42021234531) and is being reported in accordance with the reporting guidance provided in the Preferred
Reporting Items for Systematic Reviews and Meta-Analyses Protocols (PRISMA-P) statement [32, 33] (see checklist in Additional file 1).

\section{Eligibility criteria Types of studies}

Randomised controlled trials (RCTs), cluster randomised controlled trials (cluster RCTs), and observational studies (such as cohort, case-control, and cross-sectional studies) will be included. Quasi-randomised controlled trials (quasi-RCTs), which are often associated with a high risk of bias, and cross-over studies will be excluded. Case reports, guidelines, protocols, and short communication will also be excluded.

\section{Population}

We will only include studies examining the older adults without dementia aged 60 years and above who reside in their own homes and receive treatment at primary care outpatient departments. We will exclude studies that involve older adults living in retirement homes or nursing homes.

\section{Types of interventions}

We will include studies that aim specifically to assess the following interventions:

1. Home visits which aim to prevent or reduce risks related to ageing

2. Home visits which utilise at least two of the following multidimensional approaches: medical, functional, psychosocial, and environmental evaluation of problems and resources, resulting in specific recommendations for solving observed problems and preventing new ones.

\section{Types of outcome measures}

Primary outcomes We will measure QoL using validated scales such as the WHO QoL Questionnaires, WHOQoL-BREF [34] and WHOQoL-OLD [35], the 19-item Control, Autonomy, Self-Realisation and Pleasure (CASP-19) questionnaire [36], the Older People's Quality of Life (OPQoL) questionnaire [37], and the 36-item Short Form Health Survey (SF-36) [38, 39].

Secondary outcomes We will also analyse the effects of home visit interventions on the incidence of falls, depression, dementia, and emergency department admissions. 


\section{Exclusion criteria}

We will exclude studies that involve follow-up visits for recent hospital discharge and studies targeting people with one specific illness.

\section{Information sources}

A comprehensive systematic electronic search will be conducted using these databases: PubMed, Ovid MEDLINE (R), the Cochrane Library, EMBASE, the Cochrane Central Register of Controlled Trials (CENTRAL), the Cumulative Index to Nursing and Allied Health Literature (CINAHL), Web of Science, Clini calTrials.gov, the metaRegister of Controlled Trials, the Turning Research into Practice (TRIP) database, Open Grey, High Wire, the National Institute for Health and Care Excellence (NICE), and the National Institutes of Health (NIH). The search will be limited to English language articles published from January 2010 onwards.

In addition, cross-referencing will be performed, whereby the reference lists of articles will be scanned for relevant studies. We will hand-search Malaysian quality initiative or health systems project reports in the libraries of the Institute for Medical Research (IMR), Institute for Health Management (IHM), Institute for Health System Research (IHSR), Institute for Public Health (IPH), and Ministry of Health, Malaysia.

\section{Search strategy}

The search strategy will be based on the key components of the research question: population, interventions, and outcomes. It will include a mix of medical subject headings $(\mathrm{MeSH})$ terms and free-text terms in the title and abstract search fields of the databases. The keywords will be related to the participants (e.g., aged, senior, older, elder, and geriatric), home care (e.g., house calls, home visits, and home care), and the outcomes (e.g., quality of life and accidental falls). Examples of the search strategy are presented in Additional file 2 .

\section{Selection of studies}

Two review authors will examine the titles and abstracts independently and will exclude all irrelevant studies. Two review authors will independently retrieve and screen the full text of potentially relevant articles and identify those that meet the eligibility criteria. These steps will be recorded in an Excel table along with the reasons for study exclusion. To avoid duplication, data will be identified from the main source. Any disagreements that arise will be resolved through discussions with a third author. A PRISMA flow chart showing details of studies included and excluded at each stage of the study selection process will be provided [33].

\section{Data extraction}

Two reviewers will independently retrieve and record data in a data extraction form. Any disagreements will be resolved through discussion with the third reviewer. The data extraction form will include the following variables:

- General information: title, first author, publication year, and country

- Methods: study design, study duration, sample size, and mean age of the sample

- Types of intervention: visitors' professional group, number of visits, length of visits

- Outcome measures:

O Primary outcome: QoL (characteristics of the scales used to measure QoL)

O Secondary outcomes: incidence of falls, depression, dementia, and emergency department admissions

\section{Quality assessment}

Two reviewers will evaluate the possible risk of bias for each study independently. Any disagreements will be discussed with the third reviewer. We will evaluate the RCT and cluster RCT articles for the methodological quality using the Cochrane risk of bias tool (RoB 2.0) [40]. We will categorise the risk of bias as low, high, or unclear in each of the following domains: allocation concealment, random sequence generation, blinding of outcome assessment, selective outcome reporting, incomplete outcome data, and other sources of bias.

The quality assessment tool for studies with diverse designs (QATSDD) [41, 42] will be utilised to assess mixed-method studies. There are 14 QATSDD evaluative indicators for quantitative studies. Each indicator will be measured on a 4-point Likert scale as follows: 0 (not at all), 1 (very slightly), 2 (moderate), and 3 (complete). The maximum score of this tool is 42 . The quality of a study is rated as 'high' if the score is over 75\%, 'good' if it is between 50 and $75 \%$, 'moderate' if it is between 25 and $50 \%$, and 'poor' if it is below $25 \%$.

\section{Data synthesis and analysis}

If the studies are sufficiently homogenous in terms of population, interventions, and outcomes, the results will be pooled, and a meta-analysis using a random-effects model will be conducted. Where possible, dichotomous data will be presented as relative risks (RRs) with 95\% confidence intervals (CIs). Continuous data will be 
expressed as mean differences (MDs) or standardised mean differences (SMDs) (when the outcome is measured using several scales or instruments) with $95 \%$ CIs [43]. If the study characteristics are substantially different, the results will be analysed in the following subgroups, if data are available:

- Participant's age: $60-79, \geq 80$

- Visitors' professional group

We will interpret the heterogeneity and variability of the included studies in relation to population, interventions, outcomes, and methods. When meta-analysis is attempted, heterogeneity will be evaluated by forest plots to assess whether the CIs overlap. In addition, heterogeneity among the included studies will be measured using the chi-square $\left(\chi^{2}\right)$ test and $I^{2}$ statistic. A small $p$ value $(p$ $<0.1$ ) for the $X^{2}$ test and an $I^{2}$ of $50 \%$ or higher indicate moderate to substantial heterogeneity [44].

If meta-analysis is not possible, a narrative will be developed to summarise differences. We will present the data in a summary table outlining the content of the included primary studies (the number of participants, study population, description of interventions), as well as the results, conclusions, and quality ranking of studies.

\section{Meta-bias(es)}

We will assess publication bias using the Tandem method. If possible, the potential for reporting bias will be further explored using a funnel plot. A linear regression test will be performed to examine the degree of publication bias. Publication bias is significant if the p-value is less than 0.1 .

\section{Confidence in cumulative evidence}

The quality of the evidence synthesised in this review will be assessed using the Grading of Recommendations Assessment, Development and Evaluation (GRADE) methodology [45]. This methodology involves the evaluation of the evidence quality for each outcome across the domains of risk of bias, consistency, directness of evidence, precision of effect estimates, and publication bias, resulting in the following grades for each outcome: high, moderate, low, or very low $[17,46]$.

\section{Discussion}

This review may serve as evidence to support effective interdisciplinary home visits that can improve healthrelated QoL among older adults. This will potentially benefit policymakers and healthcare managers in planning for an efficient resource utilisation and evidencebased policy designs catered to older adults' health. Healthcare professionals and implementers will be able to deliver health programmes and interventions suited to the needs of the older adult population. Researchers and other institutions will gain knowledge of multiple health interventions. In addition, recognising international practices will provide information to policymakers regarding strategies to improve quality of care in future.

This review has potential limitations. Our search strategy may miss sources of information available in languages other than the English language. In addition, we anticipate that the review will face challenges due to the heterogeneous nature of the study design, particularly in interventions and outcomes measures, which may limit the interpretability and comparability of results.

\section{Protocol amendments}

Any amendments to this protocol in the carrying out of this systematic review will be documented and reported in both the PROSPERO register and any subsequent publications.

\section{Dissemination plans}

The findings of this systematic review will be disseminated through publication in peer-reviewed journals and via relevant conferences. In addition, the results will also be shared with potential stakeholders, such as the Ministry of Women, Family and Community Development and the Family Health Development Division under the Ministry of Health Malaysia.

\section{Abbreviations}

Cl: Confidence interval; Cluster RCTs: Cluster randomised controlled trials; HRQoL: Health-related quality of life; MeSH: Medical subject headings; PRISMA: Preferred Reporting Items for Systematic Reviews and Meta-Analyses; QATSDD: Quality assessment tool for studies with diverse design; QoL: Quality of life; Quasi-RCTs: Quasi-randomised controlled trials; RCTs: Randomised controlled trials; WHO: World Health Organization.

\section{Supplementary Information}

The online version contains supplementary material available at https://doi. org/10.1186/s13643-021-01862-8.

Additional file 1:. PRISMA 2020 Checklist

Additional file 2:. Search strategy

\section{Acknowledgements}

We would like to express our appreciation to the Director General of Health Malaysia for his permission to publish this systematic review protocol. We would also like to thank the Director of the Institute for Health Systems Research, National Institutes of Health Malaysia for her permission to conduct this review.

\section{Authors' contributions}

Conceiving the protocol: YLT, NSAB, and ZA. Designing the protocol: YLT and NSAB. Coordinating the protocol: ZA. Designing search strategies: YLT, NSAB, and NAAMM. Writing the protocol: YLT, NSAB, RT, NHAR, and ZA. Providing general advice on the protocol: WKY. The authors read and approved the final manuscript. 


\section{Funding}

The authors declare that they have received no specific funding for this work.

\section{Availability of data and materials}

Not applicable.

\section{Declarations}

\section{Ethics approval and consent to participate}

This systematic review protocol was registered with the National Medical Research Register (NMRR-20-1810-56054), Ministry of Health Malaysia. Ethical approval was sought from the Health Medical Research Ethics Committee (MREC), Ministry of Health Malaysia, on 9 September 2020.

\section{Consent for publication}

Not applicable.

\section{Competing interests}

The authors declare that they have no competing interests.

\section{Author details}

${ }^{1}$ Institute for Health Systems Research, National Institutes of Health, Ministry of Health Malaysia, 40170 Shah Alam, Selangor, Malaysia. ${ }^{2}$ Institute of Biological Sciences, Faculty of Science, Universiti Malaya, 50603 Kuala Lumpur, Malaysia. ${ }^{3}$ General Medical Department, Hospital Kuala Lumpur, Ministry of Health Malaysia, Jalan Pahang, 50586 Kuala Lumpur, Malaysia.

Received: 5 February 2021 Accepted: 19 November 2021 Published online: 06 December 2021

\section{References}

1. World Health Organization. Ageing and health. https://www.who.int/ news-room/fact-sheets/detail/ageing-and-health. Accessed 26 Aug 2021.

2. Institute for Public Health Malaysia. National Health and Morbidity Survey 2018 (NHMS 2018): Elderly health. Vol. I: methodology and general findings. Selangor: Institute for Public Health, National Institutes of Health, Ministry of Health Malaysia; 2019. http://iku.moh.gov.my/images/IKU/ Document/REPORT/NHMS2018/NHMS2018ElderlyHealthVolume1.pdf. Accessed 10 Oct 2020

3. Department of Statistics Malaysia. Abridged life tables, Malaysia, 20172019. https://www.dosm.gov.my/v1/index.php?r=column/pdfPrev\&id= YnV4S1FyVnNzUWJIQ3F5NHVMeFY3UT09. Accessed 10 Oct 2020.

4. Sooryanarayana R, Sazlina S-G. The Malaysian National Health and Morbidity Survey (NHMS) 2018: older persons' health in Malaysia. Geriatr Gerontol Int. 2020;20(S2):5-6.

5. Borglin G, Edberg A-K, Rahm Hallberg I. The experience of quality of life among older people. J Aging Stud. 2005;19(2):201-20.

6. World Health Organization. WHOQOL-BREF: introduction, administration, scoring and generic version of the assessment. Geneva: Programme on Mental Health, World Health Organization; 1996. http://www.who.int/ mental_health/media/en/76.pdf. Accessed 5 Oct 2020

7. Patrick DL, Erickson P. Health status and health policy: quality of life in health care evaluation and resource allocation. New York: Oxford University Press; 1993.

8. Institute for Public Health Malaysia. National Health and Morbidity Survey 2018 (NHMS 2018): Elderly health. Vol. Il: elderly health findings. Selangor: Institute for Public Health, National Institutes of Health, Ministry of Health Malaysia; 2019. http://iku.moh.gov.my/images/IKU/Document/REPORT/ NHMS2018/NHMS2018ElderlyHealthVolume2.pdf. Accessed 18 Oct 2020

9. Van Malderen L, Mets T, Gorus E. Interventions to enhance the quality of life of older people in residential long-term care: a systematic review. Ageing Res Rev. 2013;12(1):141-50.

10. Gusi N, Reyes MC, Gonzalez-Guerrero JL, Herrera E, Garcia JM. Cost-utility of a walking programme for moderately depressed, obese, or overweight elderly women in primary care: a randomised controlled trial. BMC Public Health. 2008;8:231.
11. Eyigor S, Karapolat H, Durmaz B. Effects of a group-based exercise program on the physical performance, muscle strength and quality of life in older women. Arch Gerontol Geriatr. 2007;45(3):259-71.

12. Han SJ, Kim HK, Storfjell J, Kim MJ. Clinical outcomes and quality of life of home health care patients. Asian Nurs Res. 2013;7(2):53-60.

13. Bökberg C, Ahlström G, Karlsson S. Significance of quality of care for quality of life in persons with dementia at risk of nursing home admission: a cross-sectional study. BMC Nurs. 2017;16:39.

14. Tourigny A, Bédard A, Laurin D, Kröger E, Durand P, Bonin L, et al. Preventive home visits for older people: a systematic review. Can J Aging. 2015;34(4):506-23.

15. Elkan $R$, Kendrick $D$. What is the effectiveness of home visiting or homebased support for older people? Copenhagen: WHO Regional Office for Europe; 2004. http://www.euro.who.int/Document/e83105. pdf. Accessed 14 Sept 2020

16. Tøien M, Heggelund M, Fagerström L. How do older persons understand the purpose and relevance of preventive home visits? A study of experiences after a first visit. Nurs Res Pract. 2014;2014:640583.

17. Grant S, Parsons A, Burton J, Montgomery P, Underhill K, Wilson EM. Home visits for prevention of impairment and death in older adults: a systematic review. Campbell Syst Rev. 2014;10(1):1-85.

18. Bannenberg N, Førland O, Iversen T, Karlsson M, Øien H. Preventive home visits. CINCH Working Paper Series 2019;1907:52.

19. Goroncy A, Makaroff K, Trybula M, Regan S, Pallerla H, Goodnow K, et al. Home visits improve attitudes and self-efficacy: a longitudinal curriculum for residents. J Am Geriatr Soc. 2020;68(4):852-8.

20. Liimatta H, Lampela P, Laitinen-Parkkonen P, Pitkala KH. Effects of preventive home visits on health-related quality-of-life and mortality in home-dwelling older adults. Scand J Prim Health Care. 2019;37(1):90-7.

21. Liimatta HA, Lampela P, Kautiainen H, Laitinen-Parkkonen P, Pitkala KH. The effects of preventive home visits on older people's use of health care and social services and related costs. J Gerontol A Biol Sci Med Sci. 2020;75(8):1586-93.

22. Mayo-Wilson E, Grant S, Burton J, Parsons A, Underhill K, Montgomery P. Preventive home visits for mortality, morbidity, and institutionalization in older adults: a systematic review and meta-analysis. PLoS One. 2014;9(3):e89257.

23. Sommers LS, Marton KI, Barbaccia JC, J. R. Physician, nurse, and social worker collaboration in primary care for chronically ill seniors. Arch Intern Med. 2000;160(12):1825-33.

24. Counsell SR, Callahan CM, Clark DO, Tu W, Buttar AB, Stump TE, et al. Geriatric care management for low-income seniors: a randomized controlled trial. JAMA. 2007;298(22):2623-33.

25. Seiger Cronfalk B, Fjell A, Carstens N, Rosseland LMK, Rongve A, Rönnevik DH, et al. Health team for the elderly: a feasibility study for preventive home visits. Prim Health Care Res Dev. 2017;18(3):242-52.

26. Stuck AE, Egger M, Hammer A, Minder CE, Beck JC. Home visits to prevent nursing home admission and functional decline in elderly people: systematic review and meta-regression analysis. JAMA. 2002;287(8):1022-8.

27. Institute for Health Systems Research Malaysia. National Health and Morbidity Survey (NHMS) 2019: Vol. II: healthcare demand. Selangor: Institute for Health Systems Research, National Institutes of Health, Ministry of Health Malaysia; 2020. https://ihsr.moh.gov.my/images/ publication_material/NHMS2019/hcd2019_report.pdf. Accessed 26 Aug 2021

28. Ismail NR, Abdul Hamid A, Hamid NA. Domiciliary care service: factors influencing improvement in activities of daily living among stroke survivors. Home Health Care Manag Pract. 2019;32(1):45-52.

29. Sivalingam N, Lim RBL, Rampal L. Palliative care in Malaysia: the need to do much more. Med J Malaysia. 2021;76(3):279-83.

30. Sooryanarayana R, Wong NI, Ahmad NA, Razak MAA, Yusoff MFM, Chan $Y Y$, et al. An overview of the methodology and general findings from the National Health and Morbidity Survey (NHMS) 2018: older persons' health in Malaysia. Geriatr Gerontol Int. 2020;20(S2):7-15.

31. Abdul Mutalip MH, Abdul Rahim FA, Mohamed Haris H, Yoep N, Mahmud $A F$, Salleh $R$, et al. Quality of life and its associated factors among older persons in Malaysia. Geriatr Gerontol Int. 2020;20(S2):92-7.

32. Moher D, Shamseer L, Clarke M, Ghersi D, Liberati A, Petticrew M, et al. Preferred reporting items for systematic review and meta-analysis protocols (PRISMA-P) 2015 statement. Syst Rev. 2015;4(1):1. 
33. Page MJ, McKenzie JE, Bossuyt PM, Boutron I, Hoffmann TC, Mulrow CD, et al. The PRISMA 2020 statement: an updated guideline for reporting systematic reviews. BMJ. 2021;372:n71.

34. Skevington S, Lotfy M, O'Connell K, WHOQOL Group. The World Health Organization's WHOQOL-BREF quality of life assessment: psychometric properties and results of the international field trial. A report from the WHOQOL group. Qual Life Res. 2004;13(2):299-310.

35. Power M, Quinn K, Schmidt S. Development of the WHOQOL-old module. Qual Life Res. 2005;14(10):2197-214.

36. Hyde M, Wiggins R, Higgs P, Blane D. A measure of quality of life in early old age: the theory, development and properties of a needs satisfaction model (CASP-19). Aging Ment Health. 2003;7(3):186-94.

37. Bowling A. The psychometric properties of the older people's quality of life questionnaire, compared with the CASP-19 and the WHOQOL-OLD. Curr Gerontol Geriatr Res. 2009;2009:298950.

38. Ware JE, Robert HB, Allyson RD, Kathleen NW, Anita S, William HR, et al. Conceptualization and measurement of health for adults in the health insurance study: Vol. I: model of health and methodology. Santa Monica: RAND Corporation; 1980. https://www.rand.org/pubs/reports/R1987z1. html. Accessed 17 Sept 2020

39. Burholt V, Nash P. Short Form 36 (SF-36) health survey questionnaire: normative data for Wales. J Public Health. 2011;33(4):587-603.

40. Sterne JAC, Savović J, Page MJ, Elbers RG, Blencowe NS, Boutron I, et al. RoB 2: a revised tool for assessing risk of bias in randomised trials. BMJ. 2019;366:14898

41. Sirriyeh R, Lawton R, Gardner P, Armitage G. Reviewing studies with diverse designs: the development and evaluation of a new tool. J Eval Clin Pract. 2012;18(4):746-52.

42. Fenton L, Lauckner H, Gilbert R. The QATSDD: comments and critiques. J Eval Clin Pract. 2015;21:1125-8.

43. Murad MH, Wang Z, Chu H, Lin L. When continuous outcomes are measured using different scales: guide for meta-analysis and interpretation. BMJ. 2019;364:k4817.

44. Higgins JP, Altman DG, Gøtzsche PC, Jüni P, Moher D, Oxman AD, et al. The Cochrane Collaboration's tool for assessing risk of bias in randomised trials. BMJ. 2011;343:d5928.

45. Guyatt GH, Oxman AD, Schünemann HJ, Tugwell P, Knottnerus A. GRADE guidelines: a new series of articles in the Journal of Clinical Epidemiology. J Clin Epidemiol. 2011;64(4):380-2.

46. Shamseer L, Moher D, Clarke M, Ghersi D, Liberati A, Petticrew M, et al. Preferred reporting items for systematic review and meta-analysis protocols (PRISMA-P) 2015: elaboration and explanation. BMJ. 2015;349:97647.

\section{Publisher's Note}

Springer Nature remains neutral with regard to jurisdictional claims in published maps and institutional affiliations.

Ready to submit your research? Choose BMC and benefit from:

- fast, convenient online submission

- thorough peer review by experienced researchers in your field

- rapid publication on acceptance

- support for research data, including large and complex data types

- gold Open Access which fosters wider collaboration and increased citations

- maximum visibility for your research: over $100 \mathrm{M}$ website views per year

At BMC, research is always in progress.

Learn more biomedcentral.com/submissions 